\title{
A retrospective comparison of waterbirth outcomes in two United States hospital settings
}

\author{
Joanne M. Bailey PhD, CNM ${ }^{1}$ \\ | Ruth E. Zielinski PhD, $\mathrm{CNM}^{2}$ | Cathy L. Emeis PhD, $\mathrm{CNM}^{3}$ | \\ Lisa Kane Low PhD, CNM ${ }^{1,2}$ (1)
}

\author{
${ }^{1}$ Michigan Medicine, University of \\ Michigan, Ann Arbor, Michigan \\ ${ }^{2}$ School of Nursing, University of Michigan, \\ Ann Arbor, Michigan \\ ${ }^{3}$ School of Nursing, Oregon Health \& \\ Science University, Portland, Oregon \\ Correspondence \\ Lisa Kane Low, PhD, CNM, 400 North \\ Ingalls Suite 3160, Ann Arbor 48109, MI. \\ Email: kanelow@med.umich.edu
}

\begin{abstract}
Background: Water immersion during labor is an effective comfort measure; however, outcomes for waterbirth in the hospital setting have not been well documented. Our objective was to report the outcomes from two nurse-midwifery services that provide waterbirth within a tertiary care hospital setting in the United States.
\end{abstract}

Methods: This study is a retrospective, observational, matched comparison design. Data were collected from two large midwifery practices in tertiary care centers using information recorded at the time of birth for quality assurance purposes. Land birth cases were excluded if events would have precluded them from waterbirth (epidural, meconium stained fluid, chorioamnionitis, estimated gestational age $<37$ weeks, or body mass index $>40$ ). Neonatal outcomes included Apgar score and admission to the neonatal intensive care unit. Maternal outcomes included perineal lacerations and postpartum hemorrhage.

Results: A total of 397 waterbirths and 2025 land births were included in the analysis. There were no differences in outcomes between waterbirth and land birth for Apgar scores or neonatal intensive care admissions (1.8\% vs $2.5 \%)$. Women in the waterbirth group were less likely to sustain a first- or second-degree laceration. Postpartum hemorrhage rates were similar for both groups. Similar results were obtained using a land birth subset matched on insurance, hospital location, and parity using propensity scores.

Discussion: In this study, waterbirth was not associated with increased risk to neonates, extensive perineal lacerations, or postpartum hemorrhage. Fewer women in the waterbirth group sustained first- or second-degree lacerations requiring sutures.

\section{KEYWORDS}

hydrotherapy, low risk maternity care, maternal and neonatal outcomes, midwifery, physiologic birth, waterbirth

\section{1 | INTRODUCTION}

The use of water as a therapy for pain and other ailments, currently referred to as hydrotherapy, has been documented as early as the ancient civilizations of Egypt, Greece, and
Rome. ${ }^{1}$ During labor, warm water immersion is safe for both the mother and fetus and has demonstrated positive effects for maternal experience in labor including decreased epidural use, improved coping with pain, shorter labor, and a greater sense of control during the labor process. ${ }^{2-4}$ 
Although there is an overall agreement with respect to the safety and efficacy of hydrotherapy during labor, there is no consensus on the safety and benefits of waterbirth, in part because of the paucity of research. Waterbirth is the use of warm water immersion during the second stage of labor resulting in the vaginal birth of a newborn entirely under water. Waterbirth, particularly in hospital settings in the United States, is not easily accessible, in contrast to the United Kingdom and other European countries. ${ }^{5}$ Consequently, there are limited data available on the outcomes associated with waterbirth-particularly within the hospital setting. The largest study in the United States focused on outcomes of waterbirth in the home or birth center setting demonstrating no additional risks to newborns, though possible increased risks of genital tract trauma for women. ${ }^{6}$ A joint statement by the American Academy of Pediatrics (AAP) and American College of Obstetricians and Gynecologists (ACOG) published in 2014 recommended that waterbirth only take place as part of a research study protocol with full written informed consent, ${ }^{7}$ which further constrained the availability of waterbirth. The updated 2016 ACOG Committee Opinion (endorsed by AAP) removed the recommendation that waterbirth only be offered in the context of a clinical trial, but also reaffirmed the recommendation that birth occur on land. ${ }^{8}$ The American College of Nurse-Midwives' Position Statement, Hydrotherapy in Labor and Birth, recommends that women be offered evidence-based information with respect to waterbirth and that it be available for women with uncomplicated pregnancies and labors who desire waterbirth. ${ }^{9}$

Risks cited for waterbirth have primarily focused on the newborn. Case reports of complications associated with waterbirth include near-drowning/drowning of the newborn, umbilical cord avulsion, and infection. ${ }^{2,3}$ Responses to these case reports have included evidence-based or best practice recommendations for care during waterbirth. ${ }^{5,9}$ However, a meta-analysis of larger cohort studies conducted outside the United States did not demonstrate an increased risk of negative outcomes such as lower Apgar scores, infection, neonatal admission, or death for newborns. ${ }^{10}$

Similarly, the data available from cohort studies with respect to maternal outcomes indicate that waterbirth does not increase the risk for maternal infection. ${ }^{3,11}$ In a large home birth-based cohort study in the United States, the risk for any genital tract trauma was very slightly increased in the waterbirth group (50.7\% vs $49.3 \%$ for land birth), ${ }^{6}$ although other studies have reported no difference or a decrease in risk of genital tract trauma in the waterbirth group. $^{2,3}$ Although larger studies have not identified an increase in risk of postpartum hemorrhage, ${ }^{3}$ a recent retrospective cohort trial of 230 participants in three groups waterbirth $(\mathrm{N}=58)$, water in labor $(\mathrm{N}=61)$, and neither $(\mathrm{N}=111)$ found a statistically significant increase in postpartum hemorrhage in the waterbirth group $P=.045 .{ }^{11}$ Waterbirth is consistently associated with less need for pain medication and lower perception of pain; however, it is challenging to differentiate how much of this is related to hydrotherapy in labor vs waterbirth. Women who have waterbirths report increased sense of control, ability to assume more comfortable positions, and decreased sense of birth as a medical experience. ${ }^{3,11}$

To address the paucity of research focused on waterbirth in United States hospital settings, the purpose of this study was to assess the outcomes from two nurse-midwifery services that provide waterbirth within tertiary care hospital settings. Data for this analysis come from two university hospital-based practices (Site A and Site B) in the United States, similar in size, client population, and pregnancy risk profile. Both practices have specific criteria for waterbirth candidates (Table 1) and written guidelines for the conduct of waterbirth and process for tub cleaning.

\section{2 | METHODS}

The study design was retrospective and observational, comparing neonatal and maternal outcomes between births occurring in water and those that did not (referred to as land births). Data were collected from two large midwifery practices, one in the Pacific Northwest region (Site A) and one in the Midwest region of the United States (Site B). Site A includes 12 certified nurse-midwives (CNM) equaling 6.7 full-time equivalent staffing and a caseload average of 500 births per year over the past 6 years. Site B includes 11 CNMs equaling an 8.3 full-time equivalent staffing and a caseload averaging 700 births per year over the past 6 years. Both Site A and Site B draw from metropolitan communities that are predominantly white and include families with both private and public (Medicaid) insurance coverage. Before data analysis, Institutional Review Board approval was obtained and a data use sharing agreement was completed to include deidentified data from both practice sites. A waiver of written informed consent was granted. Data collection had been occurring on an ongoing basis by the nurse-midwifery practices at both sites to assess outcomes of care and for quality improvement purposes.

Waterbirth programs were initiated at both sites in 1998. A robust data collection tool was initiated in 2006 at Site B and subsequently adopted with the same variables at Site A in 2012. Data for this analysis are consequently from 2012 to 2015 at Site A and 2006 to 2015 at Site B. The data sets were merged into a single deidentified database. Only spontaneous vaginal births were considered for analysis. All cases of waterbirth were included in the analysis. Cases of land birth that would not otherwise be eligible for a waterbirth were removed from the data set, including gestational 
TA B L E 1 Eligibility criteria for waterbirth at Site A and Site B, United States, 2006-2015

\begin{tabular}{|c|c|c|}
\hline Criteria & Site A & Site B \\
\hline \multicolumn{3}{|l|}{ Eligible for Waterbirth } \\
\hline $\begin{array}{l}\text { Singleton term pregnancy in cephalic } \\
\text { presentation }\end{array}$ & $\mathrm{x}$ & $\mathrm{x}$ \\
\hline Low-risk pregnancy & $\mathrm{x}$ & $\mathrm{x}$ \\
\hline Gestational age $<37$ 0/7 wk & $\mathrm{x}$ & $\mathrm{x}$ \\
\hline $\begin{array}{l}\text { Reassuring maternal and fetal status during } \\
\text { labor }\end{array}$ & $\mathrm{x}$ & $\mathrm{x}$ \\
\hline Client agrees to exit tub if advised to do so. & $\mathrm{x}$ & $\mathrm{x}$ \\
\hline \multicolumn{3}{|l|}{ Contraindications for waterbirth } \\
\hline $\begin{array}{l}\text { Evidence of fetal compromise through fetal } \\
\text { heart tracing }\end{array}$ & $\mathrm{x}$ & $\mathrm{x}$ \\
\hline Meconium-stained fluid & $\mathrm{x}$ & $\mathrm{x}$ \\
\hline $\begin{array}{l}\text { Presence of infection-human immunode- } \\
\text { ficiency virus, active herpes simplex virus, } \\
\text { hepatitis B }\end{array}$ & $\mathrm{x}$ & $\mathrm{x}$ \\
\hline $\begin{array}{l}\text { Excessive bleeding (per practitioner } \\
\text { judgment) }\end{array}$ & $\mathrm{x}$ & $\mathrm{x}$ \\
\hline Insulin-dependent diabetes & $\mathrm{x}$ & $\mathrm{x}$ \\
\hline Impaired cardiac function $^{\mathrm{a}}$ & $\mathrm{x}$ & $\mathrm{x}$ \\
\hline Chorioamnionitis & $\mathrm{x}$ & $\mathrm{x}$ \\
\hline Suspected macrosomia & $\mathrm{x}$ & $\mathrm{x}$ \\
\hline History of shoulder dystocia & $\mathrm{x}$ & $\mathrm{x}$ \\
\hline Body mass index $>40^{\mathrm{a}}$ & $\mathrm{x}$ & $\mathrm{x}$ \\
\hline Use of fetal scalp electrode & $\mathrm{x}$ & $\mathrm{x}$ \\
\hline Concurrent use of epidural or narcotics & $\mathrm{x}$ & $x^{b}$ \\
\hline $\begin{array}{l}\text { Inability to monitor fetal heart rate per } \\
\text { protocol selected as appropriate for the } \\
\text { patient's risk category }\end{array}$ & $\mathrm{x}$ & \\
\hline Preeclampsia & $\mathrm{x}$ & \\
\hline
\end{tabular}

${ }^{\mathrm{a}}$ Cardiomyopathy; valve disease; any cardiac condition requiring high-risk obstetric care.

${ }^{\mathrm{b}}>4 \mathrm{hs}$ after administration of narcotics permissible at Site B.

age $<37$ weeks, meconium-stained amniotic fluid, epidural use, body mass index $>40$, diagnosis of chorioamnionitis, or cesarean birth.

Eligibility for waterbirth was nearly identical for both Site A and Site B, with the exception of Site A that included restrictions for patients who receive narcotic medication during their labor, or where there was an inability to monitor the fetal heart rate according to their protocol, or if there was a diagnosis of preeclampsia (Table 1).

Waterbirths and land births were categorized and analyzed by actual, not intended, place of birth. Characteristics and outcomes of waterbirth cases were compared with land birth cases using chi-square and $t$ tests. Given the observational design of this study, to control for potential confounding variables, a post hoc analysis was then undertaken using a subset of the land birth group using propensity score matching. We compared waterbirth with land birth cases on demographic characteristics: race/ethnicity (white, yes/no), parity (nulliparous, yes/no), midwifery practice (Site A/Site B), and insurance (Medicaid, yes/no). We then entered characteristics that were statistically different at the $P<.05$ level into a logistic regression model. The regression produced a propensity score variable whose values represented each individual's probability of waterbirth on the basis of the model predictors. Each waterbirth case was then matched with a land birth case with the same propensity score. Because of the large land birth sample size, we found an exact match for each of the waterbirth cases.

Neonatal outcomes included Apgar score $<7$ at 1 and 5 minutes, admission to the neonatal intensive care (NICU), and neonatal death. The data collection tool did not include rates of infection, cord avulsion, water aspiration, or hyponatremia specifically; therefore, outcomes among newborns were compared indirectly by analyzing NICU admission rates between the groups. Maternal outcomes included perineal lacerations (no sutures/first or second degree with sutures/ third or fourth degree) and postpartum hemorrhage (estimated blood loss $<500 \mathrm{~mL} / 500 \mathrm{~mL}-1000 \mathrm{~mL} />1000 \mathrm{~mL}$ ). Estimated blood loss was a visual assessment only using the techniques described by Varney et $\mathrm{al}^{12}$ as weighing or quantified measurements of blood loss were not yet standard practice at Site A or B. At the time of data collection, $500 \mathrm{~mL}$ estimated blood loss was considered a postpartum hemorrhage, with $1000 \mathrm{~mL}$ estimated blood loss considered a severe postpartum hemorrhage. ${ }^{13}$

\section{3 | RESULTS}

A total of 2422 cases of normal spontaneous vaginal births without epidural use, meconium-stained fluid, chorioamnionitis, estimated gestational age $<37$, or body mass index $>40$ were available for analysis. All women were cared for by the nurse-midwifery services at Site A or B during prenatal and intrapartum care. Waterbirths accounted for $16.4 \%$ (397) of all spontaneous births in this data set.

There were significant differences in demographics between women in the waterbirth and land birth groups (Table 2). Women in the waterbirth group had higher rates of private insurance compared with Medicaid $(78.3 \%$ vs $66.3 \%$, $P<.001)$ and were more likely to be white $(84.8 \%$ vs $71.9 \%$, $P<.001)$. There were no differences in mean age, site, body mass index, or parity. Women with a history of prior cesarean birth were less likely to have a waterbirth (1.8\% vs $6.1 \%)$.

There were no significant differences in rates of maternal group B strep colonization or gestational age at time of birth (Table 3). There was a significant difference in the following variables when comparing waterbirth to land birth: 
T A B L E 2 Demographic and obstetric history for women who experienced waterbirths compared with women who experienced land birth, United States, 2006-2015

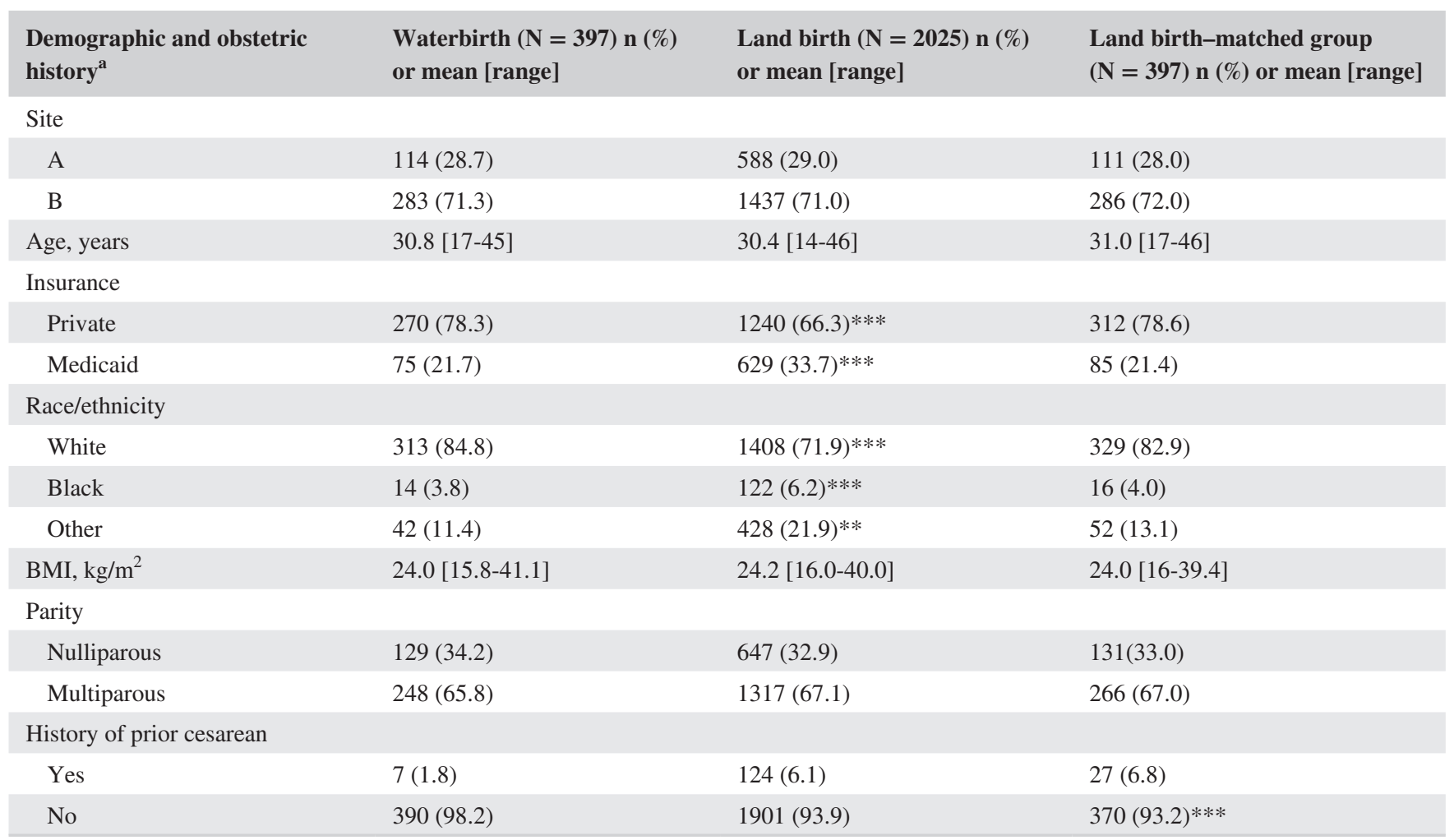

${ }^{a}$ Data for each variable not available for all participants: site group, $\mathrm{N}=2422$; age group, $\mathrm{N}=2316$; insurance group, $\mathrm{N}=2214$; race/ethnicity group, $\mathrm{N}=2327$; body mass index group, $\mathrm{N}=2157$; parity group, $\mathrm{N}=2341$; cesarean group, $\mathrm{N}=2422$.

$* P<.05$.

$* * P<.01$.

$* * * P<.001$.

induction of labor $(11.4 \%$ vs $15.3 \%, P=.04)$, augmentation of labor $(7.5 \%$ vs $11.7 \%, P=.03)$, and active management of third stage with $10 \mathrm{u}$ intramuscular oxytocin $(44.9 \%$ vs $60.5 \%$, $P<.001)$. Use of narcotics in labor also differed $(10.2 \%$ vs $18.5 \%, P<.001)$. Narcotics were never used simultaneously with water immersion. For land births, $41.3 \%$ used hydrotherapy during labor.

There were no significant differences in outcomes between waterbirth and land birth for 1-minute Apgar $<7$ (10.3\% vs $8.3 \%)$, 5-minute Apgar < 7 (0.5\% vs $0.6 \%)$, or NICU admissions ( $1.8 \%$ vs $2.5 \%$ ) (Table 4$)$. There were no perinatal deaths. Women in the waterbirth group were more likely to have an intact perineum (65.5\% vs $61.8 \%$ for land birth), similar rates of first-degree and second-degree lacerations (34.5\% vs $38.2 \%$ for land birth), and low rates of third-degree and fourth-degree perineal lacerations in both groups $(2.8 \%$ for waterbirth vs $2.9 \%$ for land birth). Postpartum hemorrhage rates were similar between groups (estimated blood loss $\geq 500 \mathrm{~mL} 9.7 \%$ for waterbirth and $7.8 \%$ for land birth and estimated blood loss $\geq 10001.3 \%$ for waterbirth and $2.7 \%$ for land birth).
Two demographic characteristics (insurance type and race/ethnicity) were identified to be significantly different between groups during the initial analysis. Therefore, we conducted a post hoc analysis, using a subset from the land birth group that matched on insurance type and race/ ethnicity, as well as site and parity, to minimize the effects of these potentially confounding variables. Neonatal and maternal outcomes remained the same in this analysis. The between-groups difference in first-degree and second-degree lacerations requiring sutures increased to $34.5 \%$ in the waterbirth group vs $41.3 \%$ in the land birth-matched comparison group. The only other significant difference between the groups was a history of cesarean (waterbirth $1.8 \%$ vs land birth $6.8 \%, P<.001)$.

\section{4 | DISCUSSION}

Results of this study indicate that waterbirth was not associated with increased risk to newborns of lower Apgar scores or NICU admission when compared with land births 
TA B L E 3 Antepartum and intrapartum characteristics for 397 women who experienced waterbirths and 2025 women who experienced land birth, United States, 2006-2015

\begin{tabular}{|c|c|c|c|}
\hline $\begin{array}{l}\text { Antepartum and intrapar- } \\
\text { tum characteristics }\end{array}$ & $\begin{array}{l}\text { Waterbirth }(\mathrm{N}=397) \mathrm{n}(\%) \text { or } \\
\text { median [range] }\end{array}$ & $\begin{array}{l}\text { Land birth }(\mathrm{N}=2025) \mathrm{n} \\
(\%) \text { or median [range] }\end{array}$ & $\begin{array}{l}\text { Land birth-matched group }(\mathrm{N}=397) \\
\text { n }(\%) \text { or median [range] }\end{array}$ \\
\hline \multicolumn{4}{|l|}{ Group B Strep } \\
\hline No & $260(68.5)$ & $1392(68.9)$ & $278(70.0)$ \\
\hline Unknown & $20(5.1)$ & $83(4.1)$ & $14(3.5)$ \\
\hline \multicolumn{4}{|l|}{ Induction } \\
\hline Yes & $45(11.4)^{*}$ & $310(15.4)$ & $61(15.4)$ \\
\hline No & $351(88.6)$ & 1705 (84.6) & 335 (84.6) \\
\hline \multicolumn{4}{|l|}{ Augmentation } \\
\hline \multicolumn{4}{|l|}{ Hydrotherapy in labor } \\
\hline Yes & $397(100.0)^{* * *}$ & $802(41.3)$ & $178(47.3)$ \\
\hline No & $0(0.0)$ & $1142(58.7)$ & $198(52.7)$ \\
\hline \multicolumn{4}{|l|}{ Narcotics in labor } \\
\hline Yes & $35(10.2)$ & $647(32.9)$ & $131(33.0)$ \\
\hline No & $308(89.8)$ & $1317(67.1)$ & $266(67.0)$ \\
\hline $\begin{array}{l}\text { Length of second stage, } \\
\text { minutes }\end{array}$ & $37.0[1-180]^{* * *}$ & $48.6[1-491]$ & $35.7[1-343]$ \\
\hline \multicolumn{4}{|c|}{ Active management of third stage } \\
\hline
\end{tabular}

${ }^{a}$ Data for each variable not available for all participants: group B Strep, $\mathrm{N}=2416$; gestational age at birth, $\mathrm{N}=2393$; induction, $\mathrm{N}=2411$; augmentation, $\mathrm{N}=2000$; hydrotherapy in labor, $\mathrm{N}=2341$; narcotics in labor, $\mathrm{N}=1639$; length of second stage, $\mathrm{N}=1435$, active management of third stage, $\mathrm{N}=2381$.

$* P<.05$.

$* * P<.01$.

$* * * P<.001$

at these hospital sites with a population cared for by certified nurse-midwives. Similarly, the risk of extensive perineal lacerations or postpartum hemorrhage was not higher for women in the waterbirth group when compared with those who experienced land births. Higher rates of intact perineum or minor lacerations not requiring sutures were seen in the waterbirth group $(65.5 \%$ vs $52.0 \%$ in the matched comparison group). Given the benefits of warm compresses to perineal outcomes,${ }^{14}$ warm water immersion in second-stage labor resulting in better perineal outcomes is not entirely unexpected. However, these results are in contrast to a prior large study comparing outcomes during home waterbirth that indicated an increased risk of genital tract trauma. ${ }^{6}$

Limitations of this study include the sample size, which is not large enough to capture differences in very rare potential complications such as neonatal death. In addition, the data collection tool did not include neonatal outcomes such as hyponatremia and cord avulsion; therefore, NICU admission was used as a proxy. In addition, the study population was limited to predominantly white women with private insurance so the results may not be generalizable to the general childbearing population. The population represented in our study is similar to recent studies identifying who is seeking waterbirth access. ${ }^{15}$ Aside from insurance type (Medicaid as a proxy for lower income status), other socioeconomic status variables were not available for analysis.

Finally, the study design was a retrospective cohort analysis, which potentially allows selection bias (by both women and midwives). Although randomized controlled trials are used as the benchmark in research, this can be problematic in the context of birth, as women are unlikely to enroll and risk randomization to the control group if 
TA B L E 4 Infant and maternal outcomes for a comparison of 397 women who experienced waterbirths and 2025 women who experienced land births, United States, 2006-2015

\begin{tabular}{|c|c|c|c|}
\hline $\begin{array}{l}\text { Infant and maternal } \\
\text { outcomes }^{\text {a }}\end{array}$ & $\begin{array}{l}\text { Waterbirth } \\
(\mathbf{N}=397) \mathbf{n}(\%)\end{array}$ & $\begin{array}{l}\text { Land birth } \\
(\mathrm{N}=2025) \mathrm{n}(\%)\end{array}$ & $\begin{array}{l}\text { Land birth-matched } \\
\text { group }(N=397) n(\%)\end{array}$ \\
\hline \multicolumn{4}{|l|}{ 1-min Apgar $<7$} \\
\hline Yes & $40(10.3)$ & $164(8.3)$ & $35(9.1)$ \\
\hline No & $350(89.7)$ & 1818 (91.7) & 349 (90.9) \\
\hline \multicolumn{4}{|l|}{ 5-min Apgar < 7} \\
\hline Yes & $2(0.5)$ & $11(0.6)$ & $5(1.3)$ \\
\hline No & $388(99.5)$ & 1955 (99.4) & $378(98.7)$ \\
\hline \multicolumn{4}{|l|}{ Admission to NICU } \\
\hline Yes & $7(1.8)$ & $51(2.5)$ & $8(2.0)$ \\
\hline No & $389(98.2)$ & 1967 (97.5) & $386(98.0)$ \\
\hline \multicolumn{4}{|l|}{ Perineal outcomes } \\
\hline $\begin{array}{l}\text { Perineum intact or no } \\
\text { sutures indicated }\end{array}$ & $260(65.5)^{*}$ & $1251(61.8)$ & $144(52.0)$ \\
\hline $\begin{array}{l}\text { First or second degree } \\
\text { lacerations with } \\
\text { sutures }\end{array}$ & $137(34.5)$ & $774(38.2)$ & $164(41.3)$ \\
\hline $\begin{array}{l}\text { Third or fourth degree } \\
\text { lacerations }\end{array}$ & $11(2.8)$ & $59(2.9)$ & $14(3.5)$ \\
\hline \multicolumn{4}{|l|}{ Estimated blood loss, $\mathrm{mL}$} \\
\hline$<500$ & $348(89.4)$ & $1760(89.5)$ & $328(85.6)$ \\
\hline $500-1000$ & $38(9.7)$ & $153(7.8)$ & $47(12.3)$ \\
\hline$>1000$ & $5(1.3)$ & $54(2.7)$ & $8(2.1)$ \\
\hline
\end{tabular}

${ }^{a}$ Data for each outcome not available for all participants: 1-min Apgar, $\mathrm{N}=2372 ; 5$-min Apgar, $\mathrm{N}=2356$; admission to NICU, $\mathrm{N}=2414$; perineal outcomes, $\mathrm{N}=2422$; estimated blood loss, $\mathrm{N}=2394$.

$* P<.05$

$* * P<.01$

$* * * P<.001$. their desire is to birth in the water. It is also noted that in the context of waterbirth, the opportunity to have a placebo or blinded measure is not possible. ${ }^{15,16}$ Randomized controlled trials (RCTs) evaluating outcomes of intended waterbirth may arguably be the next step toward evidence verifying safety and to encourage professional organizations to support wider acceptance of waterbirth in hospital settings. However, recruitment has been shown to be a barrier in attempted RCTs of birth setting; thus, similar challenges would likely be encountered for water vs land as choices during labor and birth are highly valued. ${ }^{17}$ Given the current state of the science surrounding safety of waterbirth, the suspension of access to waterbirth in the hospital setting because of the absence of a clinical trial is unwarranted. Consistent with other care options and interventions during childbirth, families can be supported to make informed choices about what best safely meets their needs.

This study was strengthened by the use of two study sites, which provided a larger sample size allowing for additional analysis using a matched comparison group and the ability to control for variables such as insurance type, race/ethnicity, and parity. Both study sites also follow waterbirth practice guidelines that optimize safety for both mother and newborn. These site-specific guidelines are consistent with those of the American College of NurseMidwives-A Model Practice Template for Hydrotherapy in Labor and Birth, which details evidence-based practices for waterbirth. ${ }^{4}$

The opportunity to have a waterbirth can be a highly desired experience for many families. The results of this study add to the evidence base supporting access to waterbirth as an extension of using hydrotherapy in the context of a low-risk pregnancy and labor for those who desire it. Evidence-based recommendations for optimizing the safety of waterbirth in the context of the hospital setting should be adhered to when the service is provided and the outcomes of care should continue to be evaluated.

\section{ACKNOWLEDGMENTS}

The authors wish to acknowledge the members of both nursemidwifery services who are committed to data collection for the purpose of ongoing quality improvement and assessment of outcomes of midwifery care. 


\section{CONFLICT OF INTEREST}

The authors report no conflict of interest.

\section{ORCID}

Joanne M. Bailey (D) https://orcid. org/0000-0003-3617-5593

Lisa Kane Low (D) https://orcid.org/0000-0002-1785-0744

\section{REFERENCES}

1. Wilson E. The Eastern, or Turkish Bath: Its History, Revival in Britain, and Application to the Purposes of Health. London: John Churchill; 1861.

2. Cluett ER, Burns E. Immersion in water in labour and birth. Cochrane Database Syst Rev. 2009;(2):CD000111.

3. Nutter E, Meyer S, Shaw-Battista J, Marowitz A. Waterbirth: an integrative analysis of peer-reviewed literature. J Midwifery Womens Health. 2014;59(3):286-319.

4. Ulfsdottir H, Saltvedt S, Georgsson S. Women's experiences of waterbirth compared with conventional uncomplicated births. Midwifery. 2019;79:1-7.

5. American College of Nurse Midwives. A model practice template for hydrotherapy in Labor and birth. J Midwifery Women's Health. 2017;62(1):120-126.

6. Bovbjerg ML, Cheyney M, Everson C. Maternal and newborn outcomes following waterbirth: the midwives alliance of North America statistics project, 2004 to 2009 cohort. J Midwifery Womens Health. 2016;61(1):11-20.

7. Committee on Obstetric Practice, American Academy of Pediatrics. ACOG Committee Opinion no. 594: Immersion in water during labor and delivery. Obstet Gynecol. 2014;123(4):912-915.

8. American college of obstetricians and gynecologists' committee on obstetric practice. Committee Opinion No. 679. Obstet Gynecol. 2016;128(5):e231-e236.

9. American College of Nurse Midwives. Position statement: hydrotherapy during labor and birth. 2016. http://www.midwife.org/ acnm/files/ccLibraryFiles/Filename/000000004048/Hydrothera
py-During-Labor-and-Birth-April-2014.pdf. Accessed September 13, 2019.

10. Taylor H, Kleine I, Bewley S, Loucaides E, Sutcliffe A. Neonatal outcomes of waterbirth: a systematic review and meta-analysis. Arch Dis Child - Fetal Neonatal Ed. 2016;101(4):F357-F365.

11. Neiman E, Austin E, Tan A, Anderson CM, Chippos EV. Outcomes of waterbirth in a US hospital-based midwifery practice: a retrospective cohort study of water immersion during labor and birth. $J$ Midwifery Womens Health. 2019;1-8.

12. King TL, Brucker MC, Jevitt C, Osborne K (Nurse-midwife). Varney's Midwifery.

13. Wormer KC, Bryant SB. Pregnancy, acute postpartum hemorrhage. 2018. http://www.ncbi.nlm.nih.gov/pubmed/29763164. Accessed November 7, 2019.

14. Aasheim V, Nilsen ABV, Reinar LM, Lukasse M. Perineal techniques during the second stage of labour for reducing perineal trauma. Cochrane Database Syst Rev. 2017;6:CD006672.

15. Sidebottom AC, Vacquier M, Simon K, et al. Who gives birth in the water? A retrospective cohort study of intended versus completed waterbirths. J Midwifery Women's Heal. 2019;64(4):403-409.

16. Oude Rengerink K, Logtenberg S, Hooft L, Bossuyt PM, Mol BW. Pregnant womens' concerns when invited to a randomized trial: a qualitative case control study. BMC Pregnancy Childbirth. 2015;15:207. https://doi.org/10.1186/s12884-015-0641-x

17. Hendrix M, Van Horck M, Moreta D, et al. Why women do not accept randomisation for place of birth: feasibility of a RCT in the Netherlands. BJOG. 2009;116(4):537-542.

How to cite this article: Bailey JM, Zielinski RE, Emeis CL, Kane Low L. A retrospective comparison of waterbirth outcomes in two United States hospital settings. Birth. 2020;47:98-104. https://doi.org/10.1111/ birt.12473 